\title{
ULTRAFAST PHOTO-TRIGGERED FIELD EMISSION CATHODES USING MASSIVE, UNIFORM ARRAYS OF NANO-SHARP HIGH-ASPECT-RATIO SILICON STRUCTURES M.E. Swanwick ${ }^{1 *}$, P. D. Keathley ${ }^{2}, F . X$. Kärtner ${ }^{2,3}$, and L. F. Velásquez-García ${ }^{l}$ \\ ${ }^{1}$ Microsystems Technology Laboratories, Massachusetts Institute of Technology, 77 Massachusetts Avenue, Cambridge, MA, 02139, USA \\ ${ }^{2}$ Dept. of Electrical Engineering and Computer Science and Research Laboratory of Electronics, Massachusetts Institute of Technology, 77 Massachusetts Avenue, Cambridge, MA, 02139, USA \\ ${ }^{3}$ Center for Free-Electron Laser Science, DESY and Dept. of Physics, University of Hamburg, Notkestralse 85, D-22607, Hamburg, Germany
}

\begin{abstract}
We report the design, fabrication, and experimental characterization of a novel ultrafast optical field emission cathode comprised of a large and dense array of nanosharp high-aspect-ratio single-crystal silicon columns $\left(>100,000\right.$ tips, 4.6-million tips. $\left.\mathrm{cm}^{-2}\right)$. The cathode emits electrons with as little as $25 \mathrm{~nJ}$ and can produce up to 2.54 $\mathrm{pC}$ electron bunches per laser pulse when interacting with $35 \mathrm{fs} 800 \mathrm{~nm}$ laser pulses with a $765 \mu \mathrm{m}$ by $80 \mu \mathrm{m}$ elliptical spot size. This is the first report of current measurements from $\mathrm{Si}$ tip arrays using photo-field emission; in addition, this is the first demonstration of a photocathode array that, depending on the energy of the laser pulses, can emit in the multi-photon or the tunnelling regime.
\end{abstract}

\section{KEYWORDS}

Coherent $\mathrm{x}$-rays, field emission, MEMS, multiplexed, photo-emission, ultrafast optics

\section{INTRODUCTION}

Fast-pulsed ( $<1 \mathrm{~ns})$, ultra-bright high-current electron sources are an enabling technology for free-electron lasers and compact coherent x-ray sources [1]. State-of-the-art ultrafast cathodes are based on the photoelectric effect, where electrons are emitted from a flat surface using ultraviolet (UV) pulses; however, these cathodes have a number of shortcomings including difficult manufacture, need for ultra-high vacuum to operate, and short lifetime [2]. Photon-triggered field emission cathodes are an attractive alternative to circumvent these issues. Strongfield electron tunnelling from solids without damage occurs when the electric field of high-intensity optical pulses interacts with field enhancing structures to lower the incident flux necessary for barrier suppression [3].

Much of the previous work on nanostructured phototriggered field emission cathodes has focused on single metal tips that are serially manufactured [4]; in this work, we used wafer-level semiconductor batch fabrication techniques to create massively multiplexed arrays of nano-sharp high-aspect-ratio silicon pillars with high uniformity ( $>100,000$ tips, 4.6-million tips. $\mathrm{cm}^{-2}$ ), resulting in greatly enhanced array electron emission. A highaspect-ratio silicon column topped by a nano-sharp tip achieves electron emission at low power by greatly enhancing the incident electric field, and the massive multiplexing of the pillars drastically increases the total current emission. In a field emitter array, a broad tip radii distribution causes severe array sub-utilization because the emission current has an exponential dependence on the field enhancement of the emitters, which is inversely proportional to the tip radii. We developed a fabrication process that attains small tip variation across the array as a result of the diffusion-limited oxidation step that sharpens the tips.

\section{DEVICE FABRICATION}

The cathodes are fabricated from a 1-10 $\Omega$-cm singlecrystal $<100>\mathrm{n}$-Si wafer. First, an array of circular features is formed on the wafer using a $250 \mathrm{~nm}$-thick chemical vapour deposited (LPCVD) silicon-rich silicon nitride film, projection optical lithography, and reactive ion etching (RIE). Then, a second array of circular features is created on top of the first array using a $500 \mathrm{~nm}$ thick plasma enhanced chemical vapour deposited (PECVD) silicon dioxide film, projection optical lithography, and RIE; each oxide feature is concentric within $0.1 \mu \mathrm{m}$ to the nitride feature right underneath. Next, the body of the pillars is etched using deep reactive ion etching (DRIE); the wafers are then cleaned and oxidized. The oxidation step thins the pillars and the nitride features act as a diffusion barrier to form the tips. The oxide and nitride films are removed using wet etchants, leaving highly uniform hexagonally packed arrays of single-crystal silicon pillars about $38 \mu \mathrm{m}$ tall and $1 \mu \mathrm{m}$ wide, topped by an ultra-sharp tip (Fig. 1). Finally, the wafers are diced into $22 \mathrm{~mm}$ squares and dipped in $2 \%$ hydrofluoric acid to remove the native oxide right before being placed in the vacuum chamber for characterization.

Prior to the photo-field emission experiments, the tip radii spread across the array was estimated by conducting

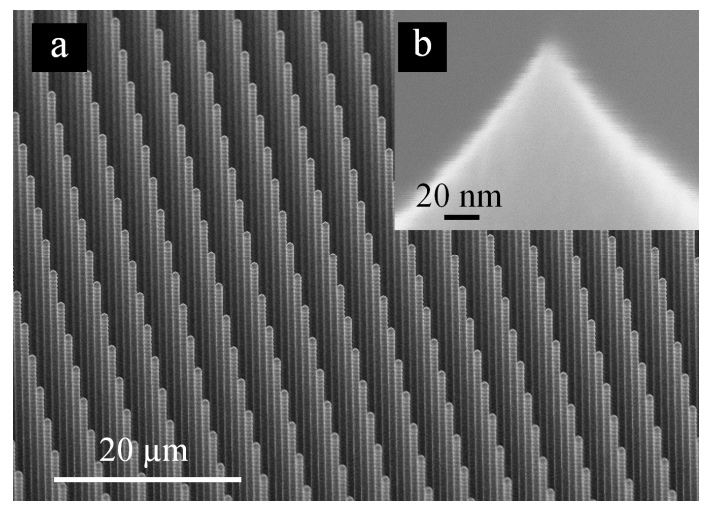

Figure 1: (a) SEM of an array of high aspect-ratio Si columns with $5 \mu \mathrm{m}$ pitch. (b) SEM close-up of a single tip. 
metrology on high-resolution FE-SEMs from 30 tips selected at random. The statistics of the tip radii result in an average radius of curvature of $4.4 \mathrm{~nm}$ with a standard deviation of $0.6 \mathrm{~nm}$ (Fig. 2), in contrast with the wide tip radii distribution commonly associated to nano-sharp silicon tip arrays [5].

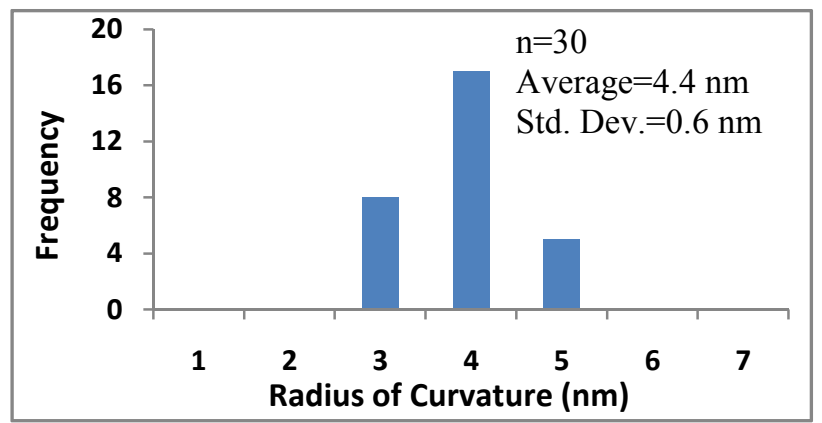

Figure 2: Histogram of the radius of curvature measurements of the tips before characterization.

\section{EXPERIMENTAL SETUP}

The arrays of nano-sharp pillars were characterized as photon-triggered cathodes in a diode configuration using a PC-controlled custom vacuum chamber with optical and electrical feedthroughs (Fig. 3). A cathode chip was placed in the vacuum chamber and pumped down to $10^{-8}$ torr pressure using a turbo-molecular pump and a dry diaphragm pump. The cathode chip was connected to ground through a picoammeter, Keithley 6485, while the anode, i.e., a 0.25 " diameter plate placed $4 \mathrm{~mm}$ above the sample, was biased at 10-500 V using a source-measuring unit (SMU) Keithley 237. The cathode was excited with $35 \mathrm{fs} 800 \mathrm{~nm}$ laser pulses at $3 \mathrm{kHz}$ repetition rate from a regeneratively amplified titanium sapphire oscillator seed. The laser pulses hit the samples at an $84^{\circ}$ grazing incidence, creating a $765 \mu \mathrm{m}$ by $80 \mu \mathrm{m}$ ellipse that bathes $\sim 2220$ tips. Data were collected using a LabView script.

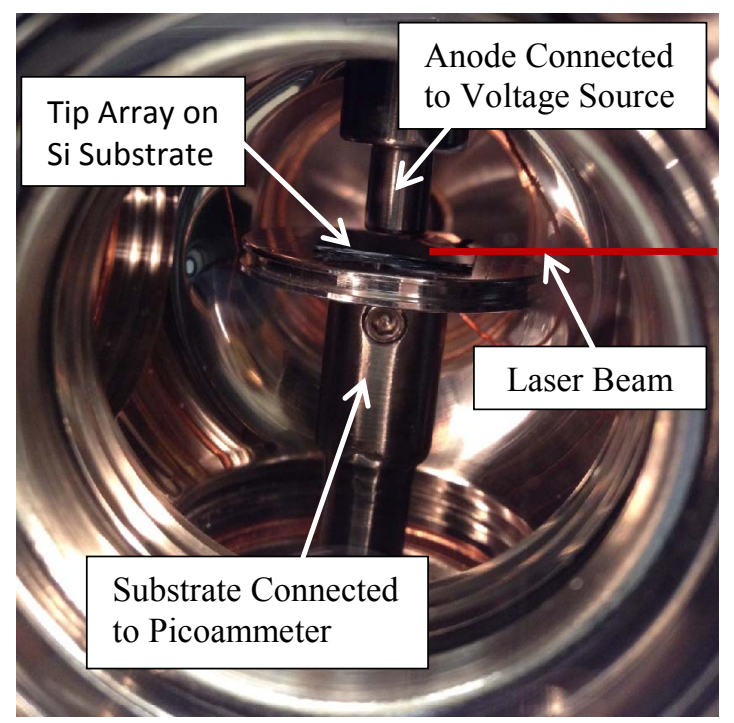

Figure 3: Experimental Set-up with an $800 \mathrm{~nm} 35$ s laser beam with $80 \mu \mathrm{m}$ spot size hitting the cathode at $84^{\circ}$.

\section{RESULTS}

In the first series of experiments, the current emitted by the nanostructured cathode was measured while the laser pulse energy was swept from $25 \mathrm{~nJ}$ to $10.8 \mu \mathrm{J}$ and the anode was biased at $10 \mathrm{~V}$. As shown in Fig. 4, the high field of the ultra-short laser pulses combined with the field enhancement of the nano-sharp high-aspect-ratio silicon tip array resulted in large current emission using small laser energies. Electron emission starts with as little as $25 \mathrm{~nJ}$ of laser pulse energy, emitting $70 \mathrm{fA}$ average current. Between $25 \mathrm{~nJ}$ and $0.1 \mu \mathrm{J}$ laser pulse energy, the data follow a 5.1-photon absorption process; the high power dependence of the emitted current on the laser pulse energy comes from the electrons oscillating back into the tip. For larger laser pulse energies the curve bends over, evidencing operation of the cathode in the tunnelling regime; this transition occurs because the electric field becomes so strong that the electrons tunnel faster than they can oscillate back into the tips [6]. If the laser pulse energy is increased to $10.8 \mu \mathrm{J}$, the pillar array emits an average current of $2.04 \mathrm{nA}(0.68 \mathrm{pC} / \mathrm{bunch})$.

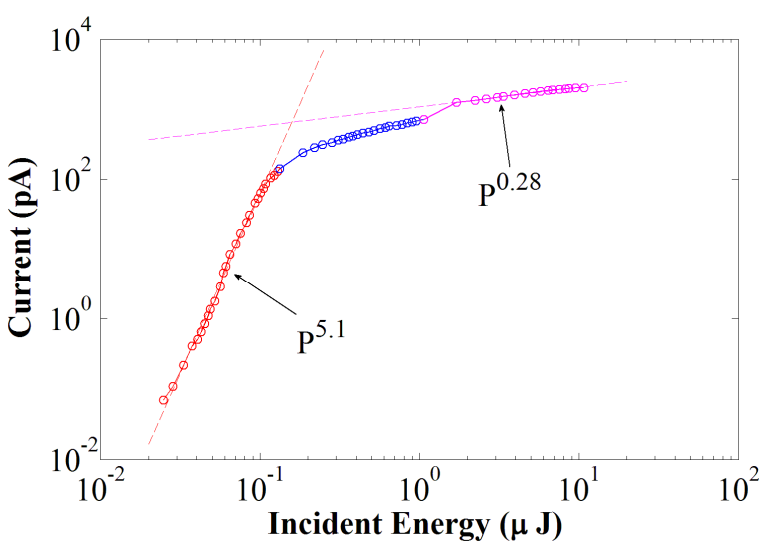

Figure 4: Log-log plot of emitted current versus incident laser pulse energy for $10 \mathrm{~V}$ anode. Red - $20 \mathrm{~dB}$ attenuator in beam line; blue - $10 \mathrm{~dB}$ attenuator in beam line; pink-without attenuator.

In a second series of experiments, the current emitted by the field emitter array was measured while the anode bias voltage was swept from $10 \mathrm{~V}$ to $500 \mathrm{~V}$ and the incident laser pulses were kept at $10.8 \mu \mathrm{J}$. The cathode chip emitted as much as $7.61 \mathrm{nA}$ average current $(2.54$ $\mathrm{pC} /$ bunch) from the $\sim 2220$ tips within the laser spot size (Fig. 5). The average number of electrons emitted per tip per cycle is then $\sim 7140$, which is the $2.54 \mathrm{pC}$ divided by $1.602 \times 10^{-7} \mathrm{pC}$ per electron divided by the number of active tips. The resultant average peak current per tip during the laser cycle is $32.7 \mathrm{~mA}$; this is calculated by dividing the $2.54 \mathrm{pC}$ by 35 fs pulse length and then dividing by the total number of tips. The electric field of the laser pulses is Gaussian and the Fowler Nordheim (FN) equation states that field emission current has an exponential dependence with respect to the electric field on the surface of the emitter tip [7]

$$
I=\alpha_{t i p} \frac{A_{F N}}{\phi t^{2}(y)} E^{2} \exp \left[\frac{-B_{F N} \phi^{3 / 2}}{E} v(y)\right]
$$




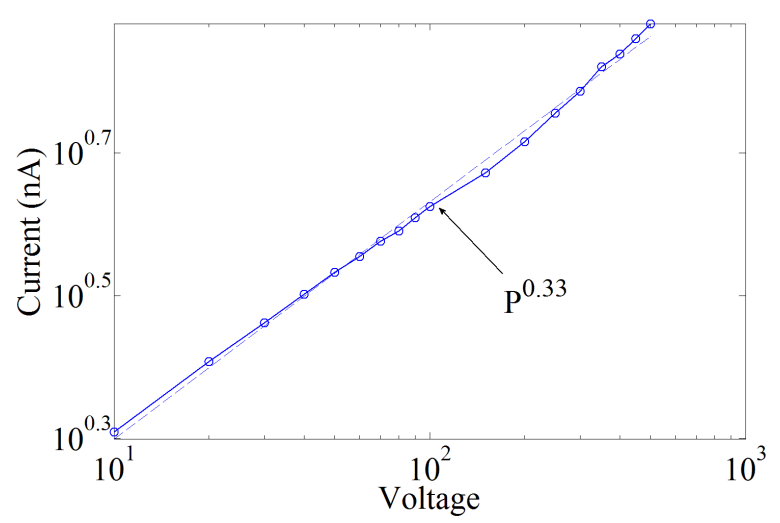

Figure 5: Log-log plot of voltage sweep at fixed incident pulse energy of $10.8 \mu \mathrm{J}$

where $I$ is the current from the tip, $\alpha_{t i p}$ is tip emitting area, $\phi$ is the workfunction at the tip/vacuum interface, $A_{F N}$ and $B_{F N}$ are constants, $t(y)$ and $v(y)$ are Nordheim elliptic functions and $E$ is the local electric field at the tip surface.

Due to the large amount of current per tip per cycle, the bend-over of the current vs. incident laser pulse (Fig. 4) and the increase in current due to increased anode bias (Fig. 5), it is important to consider space charge effects that can limit the current yield due to screening. Considering localized space charge effects in the vicinity of a single tip, Yalunin et al argue that because the electrons are emitted in a large emitting angle from a small tip, the screening effect will be greatly reduced compared to planar emitters and hence space charge effects are negligible [8]; this was experimentally observed in [6], where as many as 1000 electrons/tip were emitted with no discernible space-charge effects. Due to the fact that the electric field is mainly pointed orthogonal to the tip surface, the sharper tips used in this work should lead to even larger angular spreads due to laser acceleration, further increasing the number of electrons needed to observe local space charge effects. Regardless, the initial change of slope (red line to blue line in Fig. 4) occurs at $\sim 94$ electrons/tip/pulse -well below the emission of 1,000 electrons/tip/pulse.

Another screening effect could be caused by the formation of a virtual cathode, or electron blockade, due to the formation of a sheet of charge above the emitter array because of the close spacing of the tips and the large emitting angle of the tips. Under the sheet of charge, a uniform electric field is formed pointing toward the cathode surface; when this field is strong enough to cancel the extraction voltage field, the electrons begin to push themselves back toward the cathode, limiting the emission current. In the simplest approximation, one can calculate the critical average current for the onset of a virtual cathode as being

$$
I_{\text {crit }}=\frac{\epsilon_{0} A V f_{\text {laser }}}{D}
$$

where $V$ is the extraction voltage, $A$ is the area of the charge region (assumed to be the laser pulse spot size), $f_{\text {laser }}$ is the repetition rate of the laser, and $D$ is the separation between the emitting surface and the anode. Plugging into this simple model one finds an $I_{\text {crit }}$ of just $3.5 \mathrm{pA}$. However, this model is overly simplified, as it assumes no electron velocity and ignores aspects of the voltage near the tip such as the DC field enhancement, and electron divergence.

Other researchers have noted virtual cathode screening effects using nanostructured copper arrays for photoelectron emission [10]. By testing multi-photon emission with and without the structure, for pulses of around $5 \mathrm{x}$ longer duration (150 fs), Li et al observed virtual cathode limiting effects beginning with surface charge densities exceeding $50 \mathrm{pC} / \mathrm{mm}^{2}$ and bias fields on the order of $70 \mathrm{MV} / \mathrm{m}$; plugging these values into equation 2 results in a critical current of just $0.25 \mathrm{pA}$. However, at energies as low as $0.5 \mu \mathrm{J}$ with similar $\mathrm{Si}$ structures, spectra broader than $10 \mathrm{eV}$ were observed consistent with strong-field photoemission in the tunnelling regime [4], [9], as opposed to sub-eV bandwidths expected for multi-photon emission [10], [11]. Such contradictory observations emphasize the difficulty in describing the complicated dynamics at play with such simplified models. Further experimentation and modelling is needed to elucidate the transition between the multi-photon and strong field regimes, as well as the electron extraction dynamics after emission occurs. Fig. 5 shows the increase in emission current as the anode is swept from $10 \mathrm{~V}$ to $500 \mathrm{~V}$, resulting in a fourfold increase. There are several possible explanations for this increase including screening and space charge, as previously described, as well as DC field effects (lowering barrier) and changes to the accumulation layer on the surface of the emitters (increasing supply). A possible reason for the data shown in Fig. 5 is an increase in the electric field on the tips due to the increase in DC bias, resulting in larger electron emission. We built a $2 \mathrm{D}$ axisymmetric COMSOL Multiphysics model of a single emitter, i.e., a pillar $38 \mu \mathrm{m}$ tall and $1 \mu \mathrm{m}$ wide with tapered top that ends in a hemispherical cap with $4.5 \mathrm{~nm}$ radius of curvature. The discretization of the space surrounding the tip is very fine, with a maximum of 0.1 $\mathrm{nm}$ element size. Figure 6 shows that the local electric field around the tip is about $2.98 \mathrm{MV} / \mathrm{m}$ when an anode placed $4 \mathrm{~mm}$ from the tip is biased at $500 \mathrm{~V}$; the electric field is reduced to $59.5 \mathrm{kV} / \mathrm{m}$ for a $10 \mathrm{~V}$ anode bias. Both of these fields are orders of magnitude lower than the

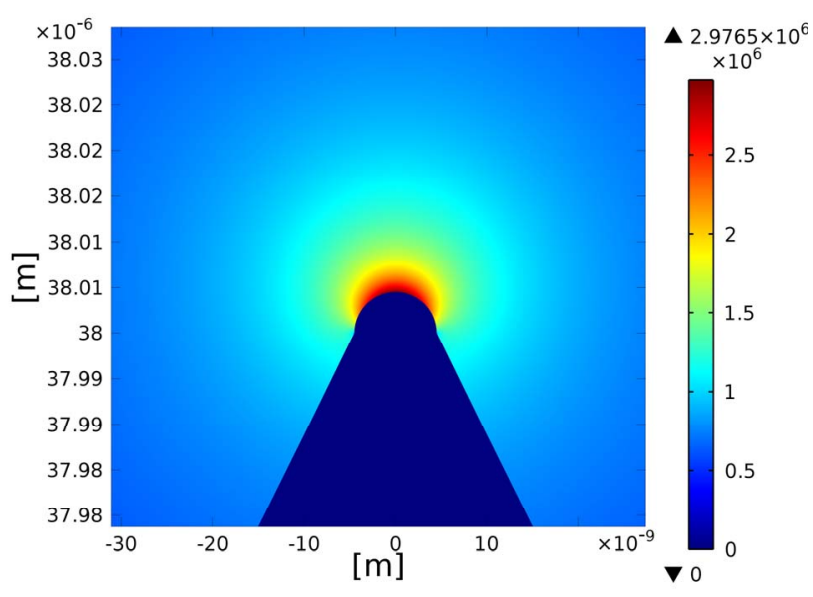

Figure 6 - Simulation of the electric field at the emitter tip from the $500 \mathrm{~V}$ anode bias (max. field of $2.98 \mathrm{MV} / \mathrm{m}$ ). 
optical field for a $10.8 \mu \mathrm{J}$ pulse of $\sim 5.8 \mathrm{GV} / \mathrm{m}$; therefore, it is unlikely that the DC anode bias plays a major effect in the electron tunnelling.

A second explanation for the data shown in Fig. 5 is the fact that n-type silicon with a vacuum interface forms an accumulation layer at the surface that generates a local electric field $E_{\max }$

$$
E_{\max }=\sqrt{\frac{2 q N_{D} \phi}{\epsilon}}
$$

where $N_{D}$ is the doping level of the cathode $\left(1 \times 10^{15} \mathrm{~cm}^{-3}\right)$, $q$ is the charge of electron, $\varepsilon$ is $1.04 \times 10^{-12} \mathrm{~F} / \mathrm{cm}$ and $\phi$ is the band gap. Using (3) we obtain a local electric field of $1.86 \times 10^{6} \mathrm{~V} / \mathrm{m}$, which is on the same order of magnitude of the electric field from the $500 \mathrm{~V}$ DC anode bias. Given that the laser pulse is Gaussian, most of the electrons are emitted at a timescale much shorter than 35 fs pulse length because of the non-linear dependence of (1). Thus, the electric field due to the anode bias increases the band bending and increases the number of electrons at the surface available to emit on these short time scales. The convolution of the all or part of the four listed possibilities is likely.

We conducted statistics on the tip radii after prolonged exposure to $10.8 \mu \mathrm{J}$ laser pulses and $500 \mathrm{~V}$ anode bias; we found an average radius of $7.8 \mathrm{~nm}$ and a standard deviation of $1.2 \mathrm{~nm}, \mathrm{n}=29$ (Fig. 7). The blunter tips with wider size spread are a result of the very high peak fields interacting with the tips. However, the performance of the arrays had no noticeable degradation at $10.8 \mu \mathrm{J}$. We are currently investigating the effect of the exposure time on the morphology of the tips and the damage threshold.

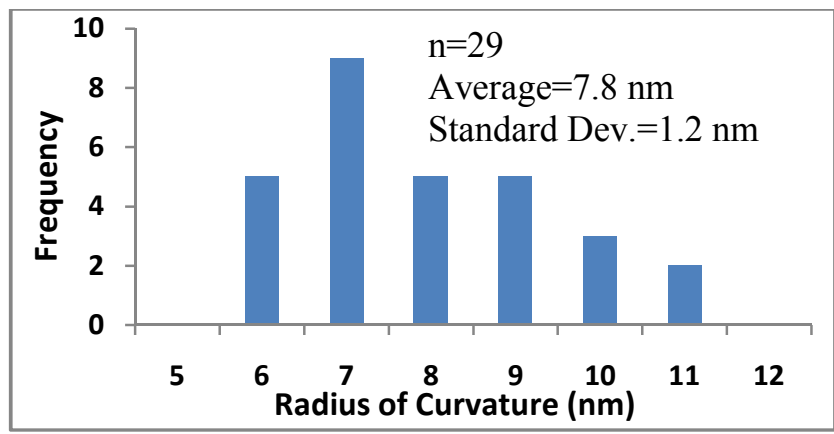

Figure 7: Histogram of the radius of curvature measurements of the post-exposure tips.

\section{ACKNOWLEDGEMENTS}

The device fabrication was done in the Microsystems Technology Laboratories, MIT. We would like the thank W. Graves, R. Hobbs and Y. Yang from MIT for the helpful discussions. This work was funded by the Defence Advanced Research Projects Agency / Microsystem Technology Office (DARPA/MTO) under contract N66001-11-1-4192 (program managers J. Mangano and D. Palmer). Any opinions, findings, and conclusions or recommendations expressed in this publication are those of the authors and do not necessarily reflect the views of the US Government and therefore, no official endorsement of the US Government should be inferred.

\section{REFERENCES}

[1] W. S. Graves, F. X. Kärtner, D. E. Moncton, and P. Piot, "Intense superradiant $\mathrm{X}$ rays from a compact source using a nanocathode array and emittance exchange," Phys. Rev. Lett., vol. 108, no. 26, p. 263904, Jun. 2012.

[2] Q. Guo, K. Takahashi, K. Saito, H. Akiyama, T. Tanaka, and M. Nishio, "Band alignment of $\mathrm{ZnTe} / \mathrm{GaAs}$ heterointerface investigated by synchrotron radiation photoemission spectroscopy," Applied Physics Letters, vol. 102, no. 9, pp. 092107092107-4, Mar. 2013.

[3] P. D. Keathley, A. Sell, W. P. Putnam, S. Guerrera, L. F. Velásquez-García, and F. X. Kartner, "Laser induced annealing dynamics of photo-electron spectra from silicon field emitter arrays", in 2012 Conference on Lasers and Electro-Optics (CLEO), $1-2,2012$.

[4] G. Herink, D. R. Solli, M. Gulde, and C. Ropers, "Field-driven photoemission from nanostructures quenches the quiver motion," Nature, vol. 483, no. 7388, pp. 190-193, Mar. 2012.

[5] M. Ding, G. Sha, and A. I. Akinwande, "Silicon field emission arrays with atomically sharp tips: turn-on voltage and the effect of tip radius distribution," IEEE Transactions on Electron Devices, vol. 49, no. 12, pp. 2333-2342, Dec. 2002.

[6] R. Bormann, M. Gulde, A. Weismann, S. V. Yalunin, and C. Ropers, "Tip-enhanced strong-field photoemission," Phys. Rev. Lett., vol. 105, no. 14, p. 147601, Sep. 2010.

[7] R. H. Fowler and L. Nordheim, "Electron emission in intense electric fields," Proc. R. Soc. Lond. A, vol. 119, no. 781, pp. 173-181, May 1928.

[8] S. V. Yalunin, M. Gulde, and C. Ropers, "Strongfield photoemission from surfaces: Theoretical approaches," Phys. Rev. B, vol. 84, no. 19, p. 195426 , Nov. 2011.

[9] P. D. Keathley, A. Sell, W. P. Putnam, S. Guerrera, L. F. Velásquez-García, and F. X. Kärtner, "Strongfield photoemission from silicon field emitter arrays," Ann. Phys., vol. 525, no. 1-2, pp. 144-150, 2013.

[10]R. K. Li, H. To, G. Andonian, J. Feng, A. Polyakov, C. M. Scoby, K. Thompson, W. Wan, H. A. Padmore, and P. Musumeci, "Surface-plasmon resonance-enhanced multiphoton emission of highbrightness electron beams from a nanostructured copper cathode," Phys. Rev. Lett., vol. 110, no. 7, p. 074801, Feb. 2013.

[11]P. Musumeci, L. Cultrera, M. Ferrario, D. Filippetto, G. Gatti, M. S. Gutierrez, J. T. Moody, N. Moore, J. B. Rosenzweig, C. M. Scoby, G. Travish, and C. Vicario, "Multiphoton photoemission from a copper cathode illuminated by ultrashort laser pulses in an $\mathrm{rf}$ photoinjector," Phys. Rev. Lett., vol. 104, no. 8, p. 084801, Feb. 2010.

\section{CONTACT}

*M. E. Swanwick, tel: +1-617-3060851; swanwick@mit.edu 